\title{
Synthesis and characterisation of larger diameter multi-walled carbon nanotubes over anodic titanium oxide template
}

\author{
I. Eswaramoorthi, Lian-Pin Hwang * \\ Institute of Atomic and Molecular Sciences, Academia Sinica and Department of Chemistry, National Taiwan University, Taipei 106, Taiwan
}

Received 9 August 2005; accepted 19 April 2006

Keywords: Carbon nanotubes; Chemical vapor deposition; Electron microscopy; Microstructure

The well known fascinating properties of carbon nanotubes (CNTs) stimulated the researchers to make extensive efforts to control their growth and properties. Different growth techniques including arc discharge [1], plasma technique [2], laser ablation [3] and pyrolysis of hydrocarbon over catalyst [4] have been used to control the position, direction and arrays of CNTs. Recently, the formation of carbon nanotubes over porous templates such as anodic aluminum oxide (AAO) inspired a new way to obtain well ordered CNTs with uniform size and shape $[5,6]$. Many reports are available on the confined formation of CNTs inside the pores of AAO with and without metal catalyst using propylene, acetylene, and polyphenyl acetylene as carbon source [6-8]. Naturally, the question arises whether other value metals such as $\mathrm{Ti}, \mathrm{Ta}, \mathrm{Nb}, \mathrm{V}, \mathrm{Hf}$ and $\mathrm{W}$ can also be used to form the CNTs inside the pore arrays fabricated by anodization process similar to AAO. Among the oxides of other value metals, titanium dioxide is considered as a promising material for photocatalyst, catalyst support and implants $[9,10]$. The catalytic activity of Lewis acid sites of $\mathrm{TiO}_{2}$ can be utilized for the growth of CNTs by pyrolysis of a suitable carbon rich hydrocarbon. Up to now no report is available on the usage of porous titanium oxide obtained by anodization, as template for the growth of CNTs. Hence, the aim of this study is to form CNTs by CVD method using acetylene as carbon source over porous titanium oxide template obtained by anodization process and to reveal the morphology and structural characteristics of the obtained CNTs using various instrumental techniques.

In this way, anodic titanium oxide (ATO) was obtained by anodization of commercially pure titanium metal in the mixture of $1 \mathrm{M} \mathrm{H}_{2} \mathrm{SO}_{4}$ and $0.5 \% \mathrm{HF}$, as electrolyte at a constant potential of $40 \mathrm{~V}$ for $72 \mathrm{~h}$. The size and morphol-

\footnotetext{
${ }^{*}$ Corresponding author. Tel.: +88622366 8287; fax: +88623620200. E-mail address: nmra@po.iams.sinica.edu.tw (L.-P. Hwang).
}

ogy of nanopores of ATO were analysed by SEM and typical top-view images are shown in Fig. 1. It is observed that more regular nanopores with diameter of $70-80 \mathrm{~nm}$ were formed. Also, the size of the nanopores is almost uniform with high density, which is beneficial for the formation of CNTs with uniform size.

The CNTs were formed over ATO template by CVD method using acetylene as carbon source with $\mathrm{Ar}$ as carrier gas at $650{ }^{\circ} \mathrm{C}$ for $2 \mathrm{~h}$, followed by annealing at $600^{\circ} \mathrm{C}$ for $15 \mathrm{~h}$. The obtained CNTs were purified by treating with $23 \% \mathrm{HF}$ for $30 \mathrm{~h}$. The features of CNTs were studied by TEM and the representative images are shown in Fig. 2. It is clearly seen that CNTs of $70-80 \mathrm{~nm}$ in diameter with a wide, empty inner space were observed. The thickness of the walls of the CNTs is found to be thin and homogeneous. Also, the length of the CNTs is in the range of several micrometers. Further, most of the CNTs are found to be straight with very few of them having branches. The open-end structure of the tip is clearly noticed (Fig. 2b). Generally, the CNTs are open-end at top side and closed end at the bottom side. Further, the walls of the CNTs are smooth with homogeneous thickness indicating the uniformity of the internal surface of nanopores. The domelike structure (Fig. 2d) of the bottom of CNTs is reflecting the structure of so-called barrier layer, a thin oxidized layer separating the porous $\mathrm{TiO}_{2}$ layer from the $\mathrm{Ti}$ metal substrate. When the CVD process is carried out at $650{ }^{\circ} \mathrm{C}$, the decomposition of acetylene in nanopores results in carbon deposition on the surface of internal pore, which constitutes the tubes and copies the three-dimensional channel structure of the template. The formation of CNTs inside the ATO pores without any metal catalyst indicates that the catalytic behavior of the internal pore surface of $\mathrm{TiO}_{2}$ plays an important role in the decomposition of acetylene, in addition to template function. Hence, it is assumed that the Lewis acid sites of the $\mathrm{TiO}_{2}$ are responsible for the catalytic activity. Since anatase and rutile forms of titanium dioxide are widely used as catalyst, 

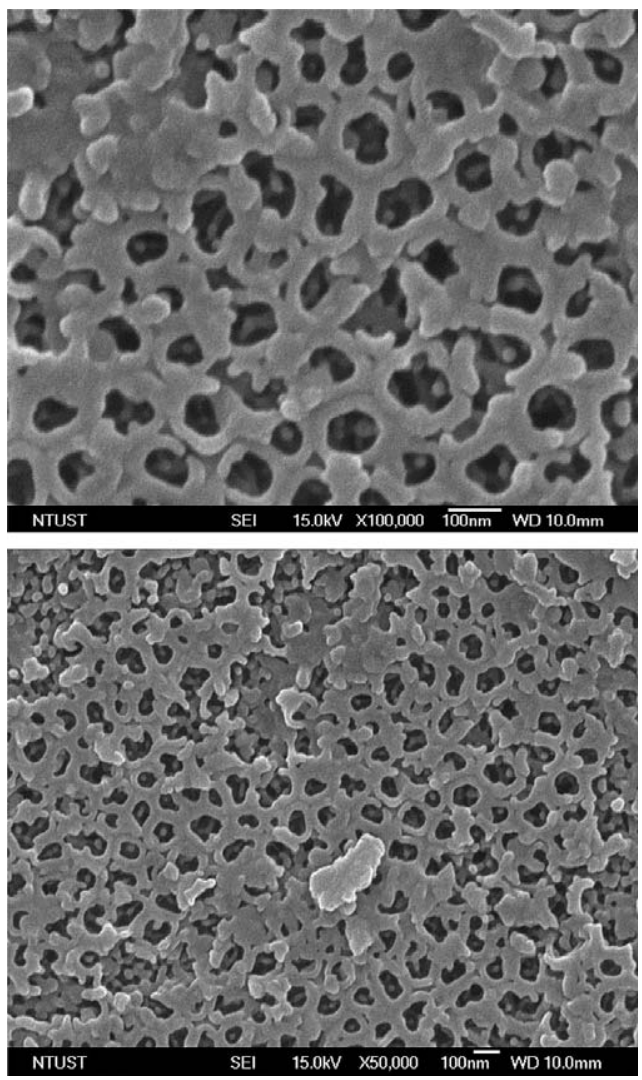

Fig. 1. Top-view SEM images of ATO template at different magnifications.
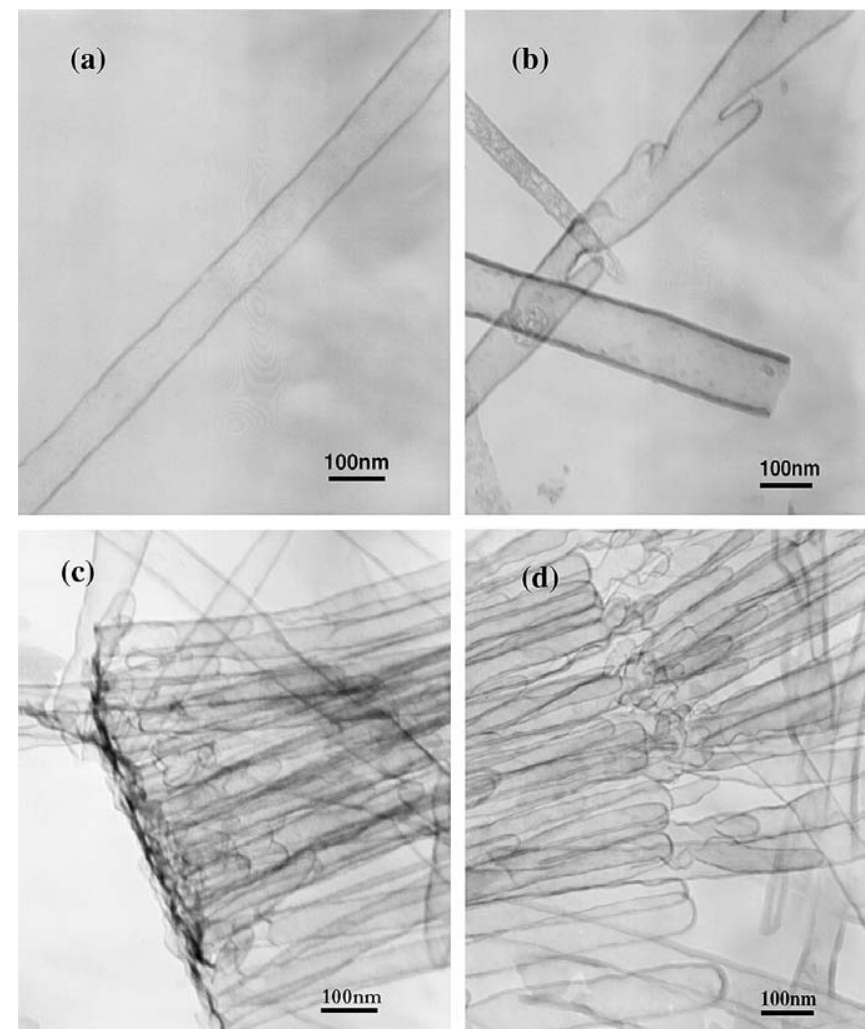

Fig. 2. TEM images showing different orientations of purified CNTs grown over ATO template. catalyst support and photocatalyst in many chemical transformations, it is expected that the strength of Lewis acid sites of porous $\mathrm{TiO}_{2}$ is high enough to catalyse the decomposition of acetylene at $650^{\circ} \mathrm{C}$. From the morphology of CNTs, it is assumed that internal surface of pores is uniform and hence uniform catalytic activity is expected. Therefore, the chance for the decomposition of acetylene is same at any point of the walls of nanopores and hence surface is uniformly covered by the carbon deposits during the CVD process, leading to the formation of carbon nanotube-like structure. Further, HRTEM measurements were also tentatively carried out to study the wall structure of the CNTs and the images are presented in Fig. 3. In spite of the amorphous background due to the specimen support film, tubes are found to be multi-walled. The wall thickness is in the range of $3 \mathrm{~nm}$ (Fig. 3a) for CNTs with deposition time of $2 \mathrm{~h}$ and it can be guessed on the image that the walls have 3-4 graphene layers with high quality nanotexture, i.e., graphenes do not show many distortions. That the CNTs are made of typical "turbostratic graphite" is likely $[11,12]$. It is interesting to note that the internal diameter of the CNTs is around $70 \mathrm{~nm}$, which is different from
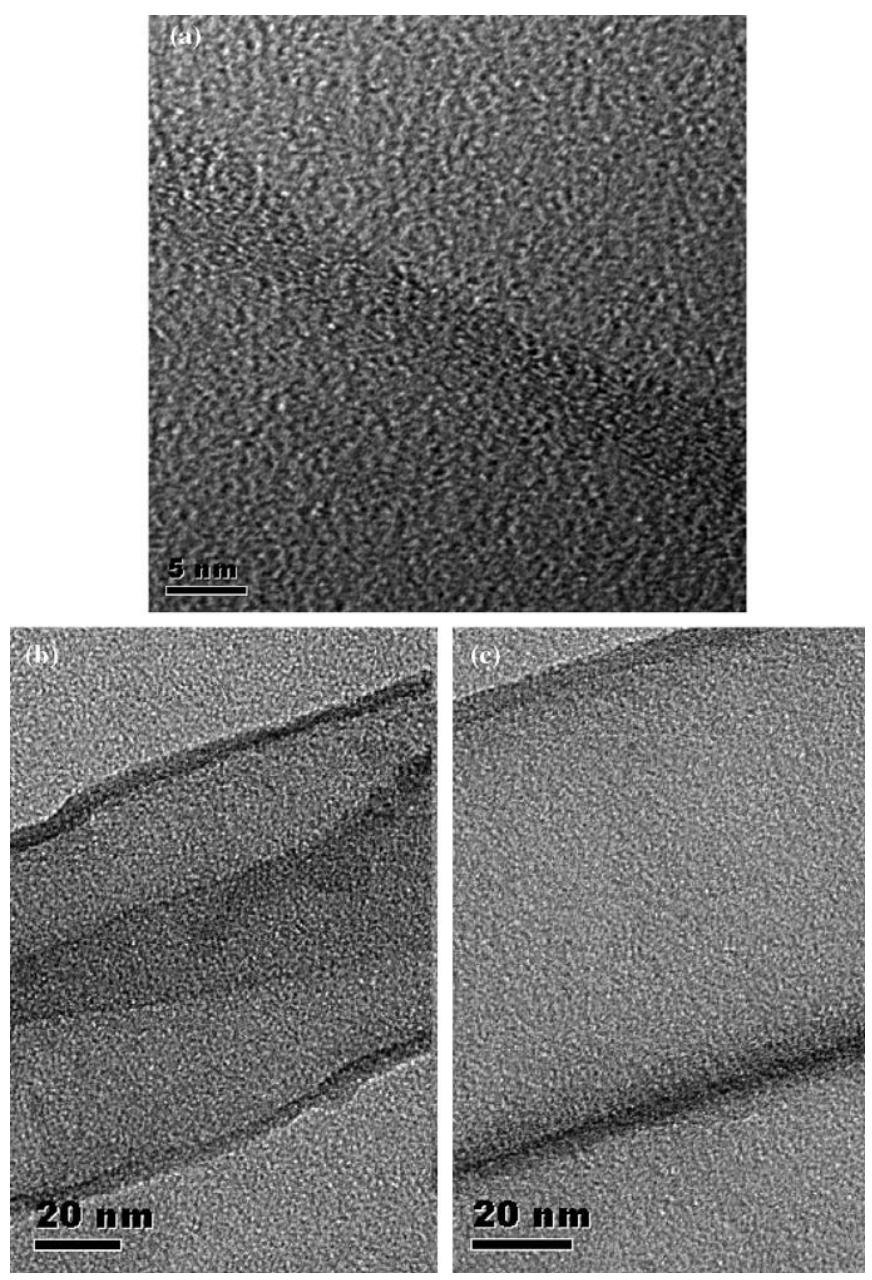

Fig. 3. HRTEM images showing the wall structure of CNTs grown with different deposition time; (a) $2 \mathrm{~h}$; (b) $4 \mathrm{~h}$ and (c) $5 \mathrm{~h}$. 
that of CNTs grown over AAO [7], which is around 15$20 \mathrm{~nm}$. This is due to the fact that only less number of graphene layers constitutes the walls of CNTs, as shown by HRTEM. The CNTs are formed on the inner surface of pores of ATO template due to the catalytic decomposition of acetylene on Lewis acid sites. The Lewis acid sites in the pores could act as a catalyst in forming of few walls of the CNTs. But, after the few walls are formed on the inner surface, the Lewis acid sites of ATO exist underneath the carbon nanotube walls. Due to this phenomenon, the Lewis acid sites of $\mathrm{TiO}_{2}$ can not act as a catalyst anymore and the catalytic decomposition of acetylene stops, typically resulting in a very thin wall as opposed to CNTs made by other methods. In order to confirm the same, CNTs were grown with deposition time of 4 and $5 \mathrm{~h}$ under similar experimental conditions and the corresponding HRTEM images are presented in Fig. $3 \mathrm{~b}$ and c, respectively. The wall thickness of CNTs in both cases is around 3-4 nm. When comparing with that of CNTs with deposition time of $2 \mathrm{~h}$ (Fig. 3a), no significant increase in wall thickness is observed. When increasing the carbon deposition time, the wall thickness of CNTs is not increased, indicating that the decomposition of acetylene is due to Lewis acid sites of $\mathrm{TiO}_{2}$ and not due to thermal decomposition.

The thermal stability of the CNTs was studied by thermo gravimetric analysis (PerkinElmer TGA) using air as carrier gas. From the TGA curve (Fig. 4), it is observed that the CNTs were stable up to $550{ }^{\circ} \mathrm{C}$ and then the decomposition starts and reaches maximum loss at $628.5^{\circ} \mathrm{C}$. At around $641^{\circ} \mathrm{C}$, almost complete weight loss is seen showing the complete decomposition of CNTs. The single sharp exothermic peak indicates that only a single phase exists in the sample. The thermal stability of the grown CNTs in air atmosphere is found to be significantly higher than the value reported $\left(480{ }^{\circ} \mathrm{C}\right)$ for CNTs grown by AAO with $\mathrm{Co}[13]$ and comparable $\left(650^{\circ} \mathrm{C}\right)$ with that of CNTs grown over FeMoMgO catalyst [14], but significantly lower than that of CNTs by arc-discharge method. The lower burning temperature of CNTs with respect to latter is related to the lesser number of layers in walls, their nanotexture quality and fact that the tubes are open-ended, meaning that graphene edges from the wall are easily accessible to the oxidizing gas.

The structure and vibrational modes of purified CNTs were analysed by XRD (X'Pert PRO-XRD) and Raman spectroscopy (Renishaw), respectively and are presented in Fig. 5. In XRD, two major peaks are observed, one is at $2 \theta=26^{\circ}$ corresponding to 002 reflexion, that relates to the stacking order in polyaromatic solids. The other small asymmetric peak around $43.5^{\circ}$ is due to 10 reflexion of turbostratic carbons. The Raman spectrum shows two strong bands at 1347 and $1592 \mathrm{~cm}^{-1}$ confirming the multi-walled nature of the CNTs. These bands are corresponding to fundamental vibration modes of $\mathrm{D}_{6 h}^{4}$ of graphite [15]. The band at $1592 \mathrm{~cm}^{-1}$ ( $\mathrm{G}$ band) corresponds to the Raman allowed optical mode $E_{2 \mathrm{~g}}$ of two-dimensional graphite, i.e., Raman active in-plane vibrational mode for an infinite or finite hexagonal network. The band at $1347 \mathrm{~cm}^{-1}$ (D band) is mainly derived from disordered carbon defects of the MWNTs, and is one of the in-plane vibrational modes.

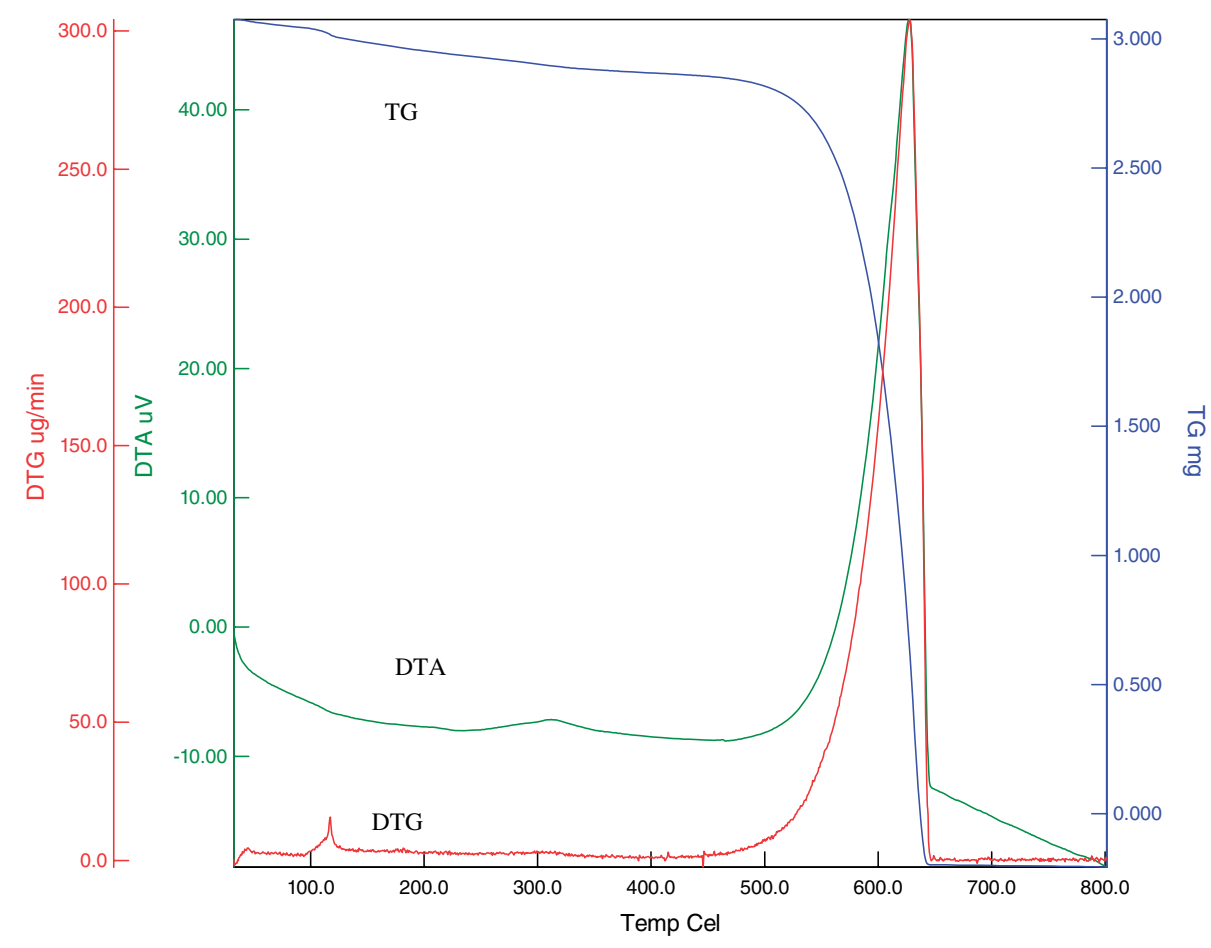

Fig. 4. TG and DTG curves of purified CNTs in air atmosphere. 

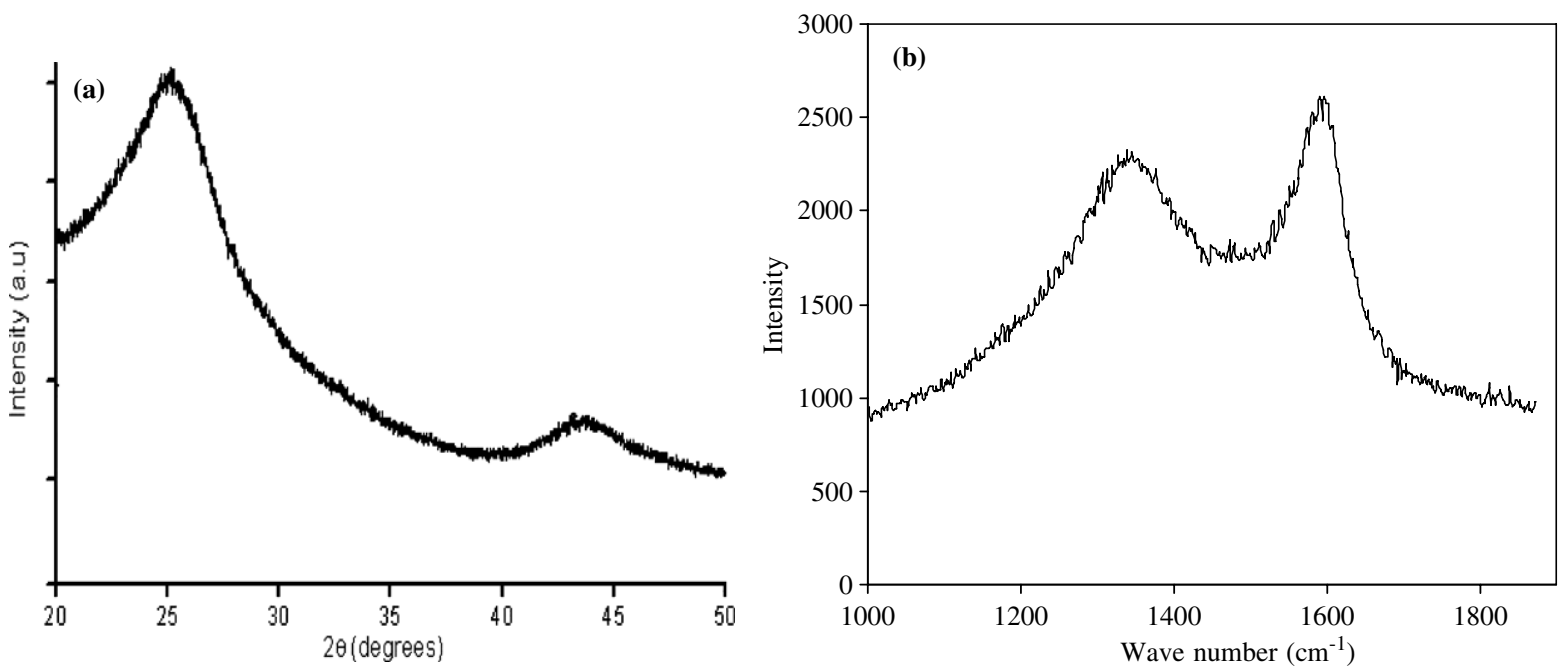

Fig. 5. XRD (a) and Raman spectroscopy (b) of CNTs grown over ATO template.

As compared to the $\mathrm{G}$ band at $1580 \mathrm{~cm}^{-1}$ for graphite, the shift in $G$ band towards higher wave number $\left(1592 \mathrm{~cm}^{-1}\right)$ as well as the $I_{\mathrm{D}} / I_{\mathrm{G}}$ value $(0.88)$ suggests a turbostratic structure, consistent with HRTEM and XRD investigations.

In summary, ordered CNTs with high nanotexture were formed, for the first time over anodic titanium oxide template and are hollow and straight. The catalytic activity of Lewis acid sites inside the pores of titanium oxide is strong enough to catalyse the decomposition of acetylene, leading to the formation of CNTs. The selective formation of CNTs over porous titanium surface could be used for the fabrication of nanofunctional devices. Also, the larger size of the CNTs favors higher metal dispersion as well as the accessibility of reactant molecules to metal particles inside the CNTs, when it is used as catalyst support in various chemical reactions.

\section{Acknowledgement}

The authors gratefully acknowledge the financial support by National Science Council of Taiwan through the Grant NSC 93-2113-M-001-047.

\section{References}

[1] Hinds BJ, Chopra N, Rantell T, Andrews R, Gavalas V, Bachas LG. Aligned multiwalled carbon nanotube membranes. Science 2004;303: $62-5$.

[2] Ebbesen T, Ajayan PM. Large-scale synthesis of carbon nanotubes. Nature 1992;358:220-2.

[3] Guo T, Nikolaev P, Thess A, Colbert DT, Smalley RE. Catalytic growth of single-walled nanotubes by laser vaporization. Chem Phys Lett 1995;243:49-54.
[4] Yacaman MJ, Yoshida MM, Rendon L, Santiesteban JG. Catalytic growth of carbon microtubules with fullerene structure. Appl Phys Lett 1993;62:202-4.

[5] Li J, Papadopoulos C, Xu JM. Highly-ordered carbon nanotube arrays for electronics applications. Appl Phys Lett 1999;75:367-9.

[6] Kyotani T, Tsai L-f, Tomita A. Preparation of ultrafine carbon tubes in nanochannels of an anodic aluminum oxide film. Chem Mater 1996;8:2109-13.

[7] Jeong S-H, Hwang H-Y, Hwang S-K, Lee K-H. Carbon nanotubes based on anodic aluminum oxide nano-template. Carbon 2004;42: 2073-80.

[8] Rajesh B, Ravindranathan Thampi K, Bonard J-M, Xanthopoulos $\mathrm{N}$, Mathieu HJ, Viswanathan B. Carbon nanotubes generated from template carbonization of polyphenyl acetylene as the support for electrooxidation of methanol. J Phys Chem B 2003;107: $2701-8$.

[9] Asahi R, Morikawa T, Ohwaki T, Aoki K, Taga Y. Visible-light photocatalysis in nitrogen-doped titanium oxides. Science 2001;293: 269-71.

[10] Albrektsson T, Branemark PI, Hansson HA, Lindstrom J. Osseointegrated titanium implants-requirements for ensuring a long-lasting, direct bone-to-implant anchorage in man. Acta Orthop Scand 1981; 52:155-70.

[11] Ivanov V, Nagy JB, Lambin P, Lucas AA, Zhang XB, Zhang XF, et al. The study of carbon nanotubules produced by catalytic method. Chem Phys Lett 1994;223:329-35.

[12] Hernadi K, Fonseca A, Nagy JB, Bernaerts D, Lucay AA Fe-Catalyzed carbon nanotube formation. Carbon 1996;34:124957.

[13] Sui YC, Acosta DR, Gonzalez-Leon JA, Bermudez A, Feuchtwanger J, Cui BZ, et al. Structure, thermal stability, and deformation of multibranched carbon nanotubes synthesized by CVD in the AAO template. J Phys Chem B 2001;105:1523-7.

[14] Jeong HJ, Kim KK, Jeong SY, Park MH, Yang CW, Lee YH. Highyield catalytic synthesis of thin multiwalled carbon nanotubes. J Phys Chem B 2004;108:17695-8.

[15] Eklund PS, Holden JM, Jishi RA. Vibrational modes of carbon nanotubes; Spectroscopy and theory. Carbon 1995;33:959-72. 